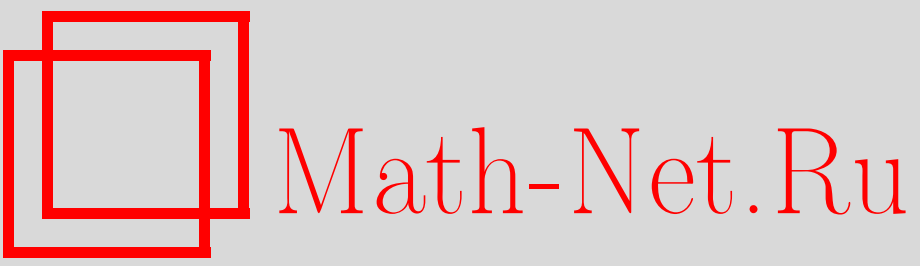

С. М. Сергеев, Свидетельство существования фазового перехода в трехмерных решеточных моделях, ТМФ, 2004, том 138, номер 3, 369-382

DOI: https://doi.org/10.4213/tmf33

Использование Общероссийского математического портала Math-Net.Ru подразумевает, что вы прочитали и согласны с пользовательским соглашением

http://www.mathnet.ru/rus/agreement

Параметры загрузки:

IP: 3.89.197.203

26 апреля 2023 г., 14:11:42 
ТЕОРЕТИЧЕСКАЯ

И МАТЕМАТИЧЕСКАЯ

ФИЗИКА

Том 138, № 3

март, 2004

(C) 2004 r.

\author{
C. М. Сергеев*
}

\title{
СВИДЕТЕЛЬСТВО СУЩЕСТВОВАНИЯ ФАЗОВОГО ПЕРЕХОДА В ТРЕХМЕРНЫХ РЕШЕТОЧНЫХ МОДЕЛЯХ
}

\begin{abstract}
Недавно было установлено, что структура собственных векторов коммутативных семейств трансфер-матриц слой-слой в трехмерных решеточных моделях описывается двумерной спиновой решеткой, обобщающей понятие одномерных спиновых цепочек. В данной работе излагаются некоторые предположения относительно связи между свойствами спиновой решетки в термодинамическом пределе и фазовой структуры трехмерных решеточных моделей. Рассматриваются два наиболее простых случая: однородная спиновая решетка, связанная с моделью Замолодчикова-Бажанова-Бакстера, и "шахматная спиновая решетка", связанная с эллиптическим решением модифицированного уравнения тетраэдров Мангазеева-Строганова. Во втором случае наблюдаются признаки существования фазового перехода.
\end{abstract}

Ключевые слова: трехмерные интегрируемые модели, модель Замолодчикова-БажановаБакстера.

\section{1. ВВЕДЕНИЕ}

Наиболее известной моделью статистической механики на кубической решетке является "двухцветная" модель Замолодчикова [1], статистическая сумма которой была вычислена Бакстером [2]. Позднее Бажанов и Бакстер нашли обобшение модели Замолодчикова на случай $N$ состояний (цветов) [3], а затем Мангазеев, Строганов и Сергеев получили вершинную формулировку этой модели [4]. Модель Замолодчикова-Бажанова-Бакстера (ЗББ) не может рассматриваться как физическая модель статистической механики, поскольку ее больцмановские веса не являются положительно определенными (более того, некоторые их них мнимы). Тем не менее главное значение статистической суммы в термодинамическом пределе предполагается положительным, так что разговор о статистической механике уместен. Однако эта механика тривиальна: как оказалось, модель ЗББ критична (см. [5]). Формулировка трехмерной решеточной модели с фазовыми явлениями представляет собой проблему, вызываюшую значительньй интерес.

* Объединенный институт ядерных исследований, Дубна, Россия; Max Planck Institut für Mathematik, Bonn, Germany. E-mail: sergeev@thsun1.jinr.ru; sergeev@mpim-bonn.mpg.de 
Относительно недавно (несколько лет назад) была сформулирована вспомогательная линейная задача для трехмерных решеточных моделей, одним из приложений которой был метод получения более широкого класса интегрируемых моделей. Например, следуюшей за моделью ЗББ в этом классе является "шахматная модель", параметризуемая в терминах эллиптических функций $[6]^{1)}$. Кроме того, вспомогательная линейная задача предлагает способ получения полного набора интегралов движения напрямую, в виде разложения некоторого операторнозначного полинома $\mathbf{J}(\lambda, \mu)$ по двум свободным комплексным параметрам $\lambda$ и $\mu$ (см. [7]).

Аналогично двумерному случаю, когда производящая функция для интегралов движения для эволюции в $(1+1)$-мерном пространстве-времени является вспомогательной трансфер-матрицей, ассоциированной с одномерной спиновой системой (т.е. со спиновой цепочкой), вспомогательная трансфер-матрица $\mathbf{J}(\lambda, \mu)$ эволюции в $(2+1)$-мерном пространстве-времени ассоциирована с двумерной спиновой системой (т.е. со спиновой решеткой)

Весьма интересно, что матрицы $\mathbf{J}(\lambda, \mu)$ не образуют коммутативное семейство. Причина заключается в том, что весь набор интегралов движения переопределен (это напоминает инвариантность центра инерции в обычной квантовой механике), однако коммутативный поднабор полон. Практически это означает, что семейство $\mathbf{J}(\lambda, \mu)$ может быть представлено одновременно в форме $(N \times N)$-матриц для $N$-цветных моделей ${ }^{2}$. Эта матричная структура позволяет вывести функциональное уравнение для спектра коммутативного поднабора [7].

Полученный недавно результат [10] заключается в том, что трансфер-матрицы типа слой-слой в трехмерных решеточных моделях пропорциональны алгебраическим дополнениям $\mathbf{J}$ как $(N \times N)$-матриц. Это наблюдение дает нам ключ к исследованию фазовой структуры трехмерных решеточных моделей. Поскольку трехмерные модели сами по себе исключительно сложны для прямого исследования (например, статистическая сумма вычислена только для модели ЗББ), то возможным выходом из положения является предсказание свойств трехмерной модели на основании исследования функционала $\mathbf{J}$ в термодинамическом пределе.

Кроме того, поскольку $\mathbf{J}(\lambda, \mu)$ содержит полный набор коммутативных операторов, то двумерная спиновая решетка по определению является вполне интегрируемой моделью и без упоминания трехмерной решеточной статистической механики, и исследование $\mathbf{J}(\lambda, \mu)$ есть отдельная важная задача. Ее важность следует из того, что для двух цветов все коммутативные операторы являются эрмитовыми, и потому система физична.

Работа построена следующим образом. Во-первых, мы сформулируем определение двумерной квадратной спиновой решетки, дадим определение $\mathbf{J}(\lambda, \mu)$ и других важных объектов, приведем функциональные уравнения для спектра $\mathbf{J}$, а затем сформулируем

\footnotetext{
1) В общей ситуации можно говорить о " $M$-шахматных моделях", параметризуемых в терминах тета-функций на алгебраической кривой рода $g=(M-1)^{2}$ (см. [7]-[9]).

2) Для сравнения, обычное семейство коммутативных трансфер-матриц может быть одновременно диагонализовано.
} 


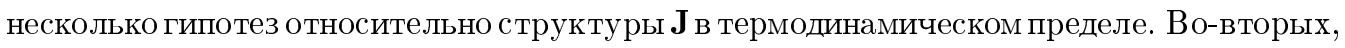
мы проверим гипотезы на примере модели ЗББ и воспроизведем ее тривиальную фазовую структуру [5]. В завершение мы исследуем простейшую шахматную решетку и получим ее нетривиальную фазовую структуру в термодинамическом пределе.

\section{2. ОПРЕДЕЛЕНИЕ КВАДРАТНОЙ СПИНОВОЙ РЕШЕТКИ}

2.1. Набор коммутативных гамильтонианов. Рассмотрим квадратную решетку размера $A \times B$ на торе. Вершины решетки можно пронумеровать парами индексов $v=(\alpha, \beta), \alpha \in \mathbb{Z}_{A}, \beta \in \mathbb{Z}_{B}$.

Пусть алгебра наблюдаемых $\mathcal{A}$ является прямым произведением $A B$ копий $\sigma$-матриц Паули:

$$
\sigma_{1}=\left(\begin{array}{cc}
0 & 1 \\
1 & 0
\end{array}\right), \quad \sigma_{2}=\left(\begin{array}{cc}
0 & i \\
-i & 0
\end{array}\right), \quad \sigma_{3}=\left(\begin{array}{cc}
1 & 0 \\
0 & -1
\end{array}\right),
$$

так что $\sigma_{j: \alpha, \beta} \in \mathcal{A}$ есть

$$
\sigma_{j: \alpha, \beta}=1 \otimes 1 \otimes \cdots \otimes \underbrace{\sigma_{j}}_{\alpha, \beta \text {-е место }} \otimes \cdots, \quad \alpha \in \mathbb{Z}_{A}, \quad \beta \in \mathbb{Z}_{B} .
$$

Матрицы Паули имеют размерность $2 \times 2$, так что согласно общепринятой терминологии мы имеем дело с двухцветным случаем.

Пусть далее $\mathbf{L}=\left\|\mathbf{L}_{v, v^{\prime}}\right\|$ - матриша размером $A B \times A B$ с операторнозначными матричными элементами

$$
\begin{aligned}
\mathbf{L}_{\alpha, \beta \mid \alpha, \beta} & =1, & \mathbf{L}_{\alpha+1, \beta+1 \mid \alpha, \beta} & =-x y c_{\alpha, \beta} \sigma_{3: \alpha, \beta}, \\
\mathbf{L}_{\alpha+1, \beta \mid \alpha, \beta} & =-x a_{\alpha, \beta} \sigma_{1: \alpha, \beta}, & \mathbf{L}_{\alpha, \beta+1 \mid \alpha, \beta} & =-y b_{\alpha, \beta} \sigma_{2: \alpha, \beta} .
\end{aligned}
$$

Здесь, вообще говоря, $a_{\alpha, \beta}, b_{\alpha, \beta}, c_{\alpha, \beta}, x, y \in \mathbb{C}$. Все остальные матричные элементы $\mathbf{L}_{v, v^{\prime}}$ - нули. Мы будем называть

$$
\lambda=x^{A}, \quad \mu=y^{B}
$$

спектральными параметрами. Благодаря ультралокальности (2) формальньй определитель $\mathbf{L}$ хорошо определен:

$$
\operatorname{det}\|\mathbf{L}\| \stackrel{\text { onp. }}{=} \mathbf{J}(\lambda, \mu)=\sum_{\alpha=0}^{A} \sum_{\beta=0}^{B} \lambda^{\beta} \mu^{\alpha} \mathbf{J}_{\alpha, \beta} .
$$

Заметим, что $\mathbf{J}$ зависит от $x, y$ через $\lambda, \mu$ (см. (4)), и к тому же $\mathbf{J}_{0,0}=1$. Пусть

$$
\mathbf{u}=\prod_{\alpha \in \mathbb{Z}_{A}} \sigma_{1: \alpha, 0}, \quad \mathbf{v}=\prod_{\beta \in \mathbb{Z}_{B}} \sigma_{2: 0, \beta} .
$$

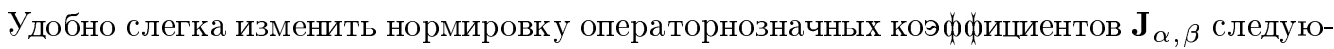
щим образом:

$$
\mathbf{J}_{\alpha, \beta}=i^{\alpha \beta} \mathbf{u}^{\beta} \mathbf{v}^{\alpha} \mathbf{t}_{\alpha, \beta} .
$$


УТВЕРЖДЕНИЕ 1. Набор $\mathbf{t}_{\alpha, \beta}$ коммутативен, $и \kappa$ тому жсе $\left[\mathbf{u}, \mathbf{t}_{\alpha, \beta}\right]=$ $\left[\mathbf{v}, \mathbf{t}_{\alpha, \beta}\right]=0$. Более того, независимыц поднабор $\mathbf{t}_{\alpha, \beta}$ и любая комбинация $\mathbf{u}, \mathbf{v}$ составляют полный набор коммутативных матрии в $\mathcal{A}$.

ДокАЗАтЕльСтво см. в работах [7]. Рассматриваемый случай соответствует локальной алгебре Вейля с $N=2$ и $q=-1$.

Запишем пару простых соотношений:

$$
\mathbf{J}(\lambda, \mu) \cdot \mathbf{u}=\mathbf{u} \cdot \mathbf{J}(\lambda,-\mu), \quad \mathbf{J}(\lambda, \mu) \cdot \mathbf{v}=\mathbf{v} \cdot \mathbf{J}(-\lambda, \mu) .
$$

ЗАмЕчАнИЕ 1 . Если параметры $a_{\alpha, \beta}, b_{\alpha, \beta}, c_{\alpha, \beta}$ вешественны, то все $\mathbf{t}_{\alpha, \beta}$ эрмитовы и поэтому являются гамильтонианами; таким образом, сформулирована физичная модель.

Гамильтонианы $\mathbf{t}_{\alpha, \beta}$ имеют сложную комбинаторную структуру для $0<\alpha<A, 0<$ $\beta<B$, и поэтому нам не удалось выделить какую-либо изящную комбинацию локальных $\sigma_{j: \alpha, \beta}$, которая напоминала бы, например, простые гамильтонианы одномерных спиновых цепочек.

2.2. Функциональное уравнение. Наряду с матрицей $\mathbf{L}$ с операторнозначными матричными элементами $(3)$ рассмотрим $\mathbb{C}$-числовую матрищу $L$ :

$$
\begin{aligned}
L_{\alpha, \beta \mid \alpha, \beta} & =1, & L_{\alpha+1, \beta+1 \mid \alpha, \beta} & =-x^{2} y^{2} c_{\alpha, \beta}^{2}, \\
L_{\alpha+1, \beta \mid \alpha, \beta} & =-x^{2} a_{\alpha, \beta}^{2}, & L_{\alpha, \beta+1 \mid \alpha, \beta} & =-y^{2} b_{\alpha, \beta}^{2} .
\end{aligned}
$$

Как и раньше, мы будем использовать определитель $L$ :

$$
\operatorname{det}\|L\|=J\left(\lambda^{2}, \mu^{2}\right)
$$

УТВЕРЖДЕНИЕ 2. Абелева алгебра $\mathbf{t}_{\alpha, \beta}$ производится разложением уравнения

$$
\underset{\mathbf{u}, \mathbf{v}}{\operatorname{det}}\|\mathbf{J}(\lambda, \mu)\|=J\left(\lambda^{2}, \mu^{2}\right)
$$

по $\lambda^{2}, \mu^{2}$, где $\operatorname{det}_{\mathbf{u}, \mathbf{v}}$ есть определитель $\mathbf{J}$ как $(2 \times 2)$-матрицв в блоке $\mathbf{u}, \mathbf{v}$. С другой сторонь, это есть функциональное уравнение для спектра $\mathbf{t}_{\alpha, \beta}$.

ДокАЗАТЕЛЬСТво см. в работах [7].

ЗАМЕЧАНИЕ 2. $\mathbf{J}(\lambda, \mu)$ - производящий функционал для интегралов движения для трехмерной решеточной модели. С помошью вершинных весов трехмерной решеточной модели можно построить вырожденную матрицу $\mathbf{Q}_{0}(\lambda, \mu)$ такую, что

$$
\begin{aligned}
\mathbf{Q}_{0}(\lambda, \mu) \cdot \mathbf{t}_{\alpha, \beta} & =\mathbf{t}_{\alpha, \beta} \cdot \mathbf{Q}_{0}(\lambda, \mu) \quad \text { для всех } \alpha, \beta, \\
\mathbf{Q}_{0}(\lambda, \mu) \cdot \mathbf{u} & =\mathbf{u} \cdot \mathbf{Q}_{0}(\lambda,-\mu), \quad \mathbf{Q}_{0}(\lambda, \mu) \cdot \mathbf{v}=\mathbf{v} \cdot \mathbf{Q}_{0}(-\lambda, \mu),
\end{aligned}
$$

а обычная трансфер-матрица типа слой-слой есть

$$
\mathbf{Q}\left(\lambda^{2}, \mu^{2}\right)=\mathbf{Q}_{0}(\lambda, \mu)+\mathbf{Q}_{0}(\lambda,-\mu)+\mathbf{Q}_{0}(-\lambda, \mu)+\mathbf{Q}_{0}(-\lambda,-\mu) .
$$


Наиболее важное свойство $\mathbf{Q}_{0}$ заключается в том, что она удовлетворяет уравнению Бакстера:

$$
\mathbf{J}(\lambda, \mu) \mathbf{Q}_{0}(\lambda, \mu)=0,
$$

так что $\lambda, \mu$ суть не произвольные числа, а координаты точки на "квантовой спектральной кривой" $J\left(\lambda^{2}, \mu^{2}\right)=0$. Матрица $\mathbf{Q}_{0}$ пропорциональна матрице алгебраических дополнений вырожденной $\mathbf{J}$. Заметим, что в трехмерной модели $x$ и $y$ (и следовательно, $\lambda$ и $\mu$ ), вообше говоря, комплексны, так что физическая интерпретация остается под вопросом. Подробности можно найти в работе [10].

Поскольку $\mathbf{u}^{2}=\mathbf{v}^{2}=1$, можно разложить

$$
\mathbf{J}(\lambda, \mu)=T_{0,0}\left(\lambda^{2}, \mu^{2}\right)+\lambda \mathbf{u} T_{1,0}\left(\lambda^{2}, \mu^{2}\right)+\mu \mathbf{v} T_{0,1}\left(\lambda^{2}, \mu^{2}\right)+i \lambda \mu \mathbf{u v} T_{1,1}\left(\lambda^{2}, \mu^{2}\right),
$$

так что

$$
T(\lambda, \mu)=T_{0,0}\left(\lambda^{2}, \mu^{2}\right)+\lambda T_{1,0}\left(\lambda^{2}, \mu^{2}\right)+\mu T_{0,1}\left(\lambda^{2}, \mu^{2}\right)+i \lambda \mu T_{1,1}\left(\lambda^{2}, \mu^{2}\right)
$$

производит полный набор интегралов движения. В частности, $\mathbf{J}(\lambda, \mu)=T(\lambda \mathbf{u}, \mu \mathbf{v})$ с точностью до упорядочивания и

$$
\begin{aligned}
\underset{\mathbf{u}, \mathbf{v}}{\operatorname{det}}\|\mathbf{J}\| & =T_{0,0}^{2}-\lambda^{2} T_{1,0}-\mu^{2} T_{0,1}-\lambda^{2} \mu T_{1,1}= \\
& =\frac{1}{2}(T(\lambda, \mu) T(-\lambda,-\mu)+T(-\lambda, \mu) T(\lambda,-\mu)) .
\end{aligned}
$$

2.3. Термодинамический предел. Как обычно, термодинамический предел понимается как главная асимптотика при $A, B \rightarrow \infty$.

ГИПоТЕЗА 1. Если в термодинамическом пределе асимптотически

$$
J \rightarrow e^{A B \mathfrak{f}}, \quad \mathfrak{f} \in \mathbb{R}, \quad \mathfrak{f}>0,
$$

то максимальные собственные значения всех

$$
|T( \pm \lambda, \pm \mu)|,\left|T_{0,0}\right|,\left|T_{1,0}\right|,\left|T_{0,1}\right|,\left|T_{1,1}\right|
$$

стремятся $к e^{A B \mathfrak{f} / 2}$. Конечно же, состояния, на которых $T_{k, l}$ принимают свои максимальные значения, должны быть различны.

Видимо, эта гипотеза справедлива даже при $J\left(\lambda^{2}, \mu^{2}\right)=0$ для конечных $A, B$. В этом случае $\mathfrak{f}$ все равно можно определить. В результате матричные элементы $\mathbf{Q}_{0}(\lambda, \mu)$ в замечании 2 должны быть также пропорциональны $e^{A B \mathfrak{f} / 2}$.

ГиПотеЗА 2. Асимптотика $\mathfrak{f}$ в случае $J\left(\lambda^{2}, \mu^{2}\right)=0$ coдержит все главные трансцендентные вырахения для свободной энергии трехмерных решеточных моделей.

Ниже мы покажем, что эта гипотеза пришла из исследования модели ЗББ. 
ГИПотеЗА 3. Аналитическая структура $\mathfrak{f}$ отражает фазовую структуру набора $\mathbf{t}_{\alpha, \beta}$ в термодинамическом пределе.

Интересно отметить, что модель свободных фермионов - хорошо известная модель статистической механики на двумерной решетке - имеет статистическую сумму, также представимую в виде определителя большой матрицы (см., например, [11]). Структура нашего определителя $J$ немного другая. Основное же различие заключается в том, что определитель модели свободных фермионов не может быть нулем для конечного объема $A B$, в то время как в нашем случае $J\left(\lambda^{2}, \mu^{2}\right)=0$ есть наиболее интересный класс.

2.4. Изменение обозначений. Ниже мы будем исследовать асимптотическое значение $J$. Для краткости перестанем писать знаки второй степени, выбрав следуюшие окончательные обозначения:

$$
\begin{aligned}
L_{\alpha, \beta \mid \alpha, \beta} & =1, & L_{\alpha+1, \beta+1 \mid \alpha, \beta} & =-x y c_{\alpha, \beta}, \\
L_{\alpha+1, \beta \mid \alpha, \beta} & =-x a_{\alpha, \beta}, & L_{\alpha, \beta+1 \mid \alpha, \beta} & =-y b_{\alpha, \beta},
\end{aligned}
$$

$\lambda=x^{A}, \mu=y^{B}, J(\lambda, \mu)=\operatorname{det}\|L\|$, и

$$
\mathfrak{f}=\lim _{A, B \rightarrow \infty} \frac{1}{A B} \ln \operatorname{det}\|L\| .
$$

\section{3. ОДНОРОДНЫЙ СЛУЧАЙ}

Начнем исследование $J$ и $\mathfrak{f}$ с простейшего однородного случая:

$$
a_{\alpha, \beta}=1, \quad b_{\alpha, \beta}=1, \quad c_{\alpha, \beta}=c .
$$

Тогда в результате преобразования Фурье можно легко вычислить

$$
J(\lambda, \mu)=\prod_{\alpha=0}^{A-1} \prod_{\beta=0}^{B-1}\left(1-x e^{2 \pi i \alpha / A}-y e^{2 \pi i \beta / B}-x y c e^{2 \pi i(\alpha / A+\beta / B)}\right),
$$

где $x^{A}=\lambda_{\text {и }} y^{B}=\mu$. Пусть

$$
\chi_{1}(x, y)=1-x-y-x y c .
$$

Тогда в термодинамическом пределе

$$
\mathfrak{f}=\frac{1}{(2 \pi)^{2}} \iint_{0}^{2 \pi} d \phi d \phi^{\prime} \ln \left|\chi_{1}\left(x e^{i \phi}, y e^{i \phi^{\prime}}\right)\right| .
$$

Заметим для начала, что $\mathfrak{f}$ зависит от $|x|$ и $|y|$. Для физического режима $c$ должна быть вешественной и положительной ${ }^{3)}$. Условие $J(\lambda, \mu)=0$ соответствует случаю, когда

\footnotetext{
3) Вообще говоря, $c \in \mathbb{R}$ есть необходимое условие для $\mathfrak{f} \in \mathbb{R} ; c>0$ следует из замечания 1 и благодаря смене обозначений (формула (20)).
} 
один из множителей в произведении (23) есть нуль, т.е. $\chi_{1}(x, y)=0$ в одной или двух точках на единичных окружностях $x=|x| e^{i \phi}, y=|y| e^{i \phi^{\prime}}$. Поскольку логарифмическая сингулярность интегрируема, формула (25) все равно справедлива.

В режиме, когда $\chi_{1}(x, y)$ имеет два нуля, без ограничения обшности можно запараметризовать

$$
|x|=\frac{\sin \beta_{2}}{\sin \beta_{1}}, \quad|y|=\frac{\sin \beta_{3}}{\sin \beta_{1}}, \quad c=\frac{\sin \beta_{0} \sin \beta_{1}}{\sin \beta_{2} \sin \beta_{3}},
$$

где по определению

$$
\beta_{0}=\pi-\beta_{1}-\beta_{2}-\beta_{3} .
$$

Нули $\chi_{1}$ есть

$$
\chi_{1}\left(\frac{1-e^{-2 i \beta_{2}}}{1-e^{2 i \beta_{1}}}, \frac{1-e^{2 i \beta_{3}}}{1-e^{-2 i \beta_{1}}}\right)=\chi_{1}\left(\frac{1-e^{2 i \beta_{2}}}{1-e^{-2 i \beta_{1}}}, \frac{1-e^{-2 i \beta_{3}}}{1-e^{2 i \beta_{1}}}\right)=0 .
$$

Благодаря очевидной симметрии

$\mathfrak{f}\left(\beta_{1}, \beta_{2}, \beta_{3}\right)=\mathfrak{f}\left(\pi+\beta_{1}, \beta_{2}, \beta_{3}\right)=\mathfrak{f}\left(\beta_{1}, \pi+\beta_{2}, \beta_{3}\right)=\mathfrak{f}\left(\beta_{1}, \beta_{2}, \pi+\beta_{3}\right)=\mathfrak{f}\left(-\beta_{1},-\beta_{2},-\beta_{3}\right)$

любой набор $\beta_{1}, \beta_{2}, \beta_{3}$ может быть приведен к каноническому:

$$
0 \leqslant \beta_{1}+\beta_{2}<\pi, \quad 0 \leqslant \beta_{1}+\beta_{3}<\pi, \quad 0 \leqslant \beta_{2}+\beta_{3}<\pi .
$$

В режиме (30)

$$
\mathfrak{f}=\mathfrak{I}\left(\beta_{0}\right)+\mathfrak{I}\left(\beta_{1}\right)+\mathfrak{I}\left(\beta_{2}\right)+\mathfrak{I}\left(\beta_{3}\right)-\ln \left|2 \sin \beta_{1}\right|,
$$

где функция $\mathfrak{I}$ связана с дилогарифмом:

$$
\Im(\beta) \stackrel{\text { oпp. }}{=} \frac{1}{\pi} \int_{0}^{\beta} \alpha \operatorname{ctg} \alpha d \alpha, \quad-\pi<\beta<\pi,
$$

или, эквивалентно,

$$
\Im(\beta)=\frac{\beta}{\pi} \ln |2 \sin \beta|+\sum_{m=1}^{\infty} \frac{\sin 2 m \beta}{2 \pi m^{2}}
$$

с главными используемыми свойствами

$$
\mathfrak{I}(\beta)+\mathfrak{I}(-\beta)=0, \quad \mathfrak{I}(\beta)+\Im(\pi-\beta)=\ln |2 \sin \beta| .
$$

Ответ включает как физический, так и не физический режимы для $c$.

Трехмерная решеточная модель, соответствуюшая однородным коэффициентам (22), есть модель ЗББ [1]-[3]. Параметры $\beta_{j}$ - линейные эксцессы сферического треугольника в бакстеровской параметризации [2], а выражение (31) очень близко́ к формуле Бакстера для плотности статистической суммы

$$
\ln z=\sum_{j=0}^{3}\left(\Im\left(\beta_{j}\right)-\frac{\beta_{j}}{2 \pi} \ln \left(2 \sin \beta_{j}\right)\right)
$$


в "геометрическом" режиме $0 \leqslant \beta_{j}<\pi, j=0,1,2,3$ (см. [2]). Это наблюдение аргументирует гипотезу 2.

Режим, когда уравнение $\chi_{1}(x, y)=0$ не имеет решений на единичных окружностях (или имеет только одно решение, например $\chi_{1}(1,1)=0$ ), может быть параметризован посредством

$$
x=\frac{\operatorname{sh} \beta_{2}}{\operatorname{sh} \beta_{1}}, \quad y=\frac{\operatorname{sh} \beta_{3}}{\operatorname{sh} \beta_{1}}, \quad x y c=\frac{\operatorname{sh}\left(\beta_{1}+\beta_{2}+\beta_{3}\right)}{\operatorname{sh} \beta_{1}}
$$

с $\beta_{j} \in \mathbb{R}(\bmod i \pi)$. Эта параметризация бесполезна, поскольку теперь

$$
\mathfrak{f}=\ln \max (1,|x|,|y|,|x y c|) .
$$

ЗАмЕчаниЕ 3. Функция $\mathfrak{f}$, заданная формулой (37), не является гладкой, однако причина этого тривиальна. Для конечных $A, B$ и $\lambda=x^{A}, \mu=y^{B}$ набор целых пар $(\alpha, \beta)$ полинома $J=\sum_{\alpha, \beta} \lambda^{\beta} \mu^{\alpha} J_{\alpha, \beta}$ назьвается многоугольником Ньютона. Например, углы этого многоугольника даются выражением

$$
J(\lambda, \mu)=1+\cdots+(-)^{B} x^{A B}+\cdots+(-)^{A} y^{A B}+(-)^{A+B}(x y c)^{A B} .
$$

Формула (37) просто описывает режимы, когда один из углов многоугольника Ньютона доминирует.

Поскольку однородная модель связана с моделью Замолодчикова, которая критична [5], признаки фазового перехода в выражениях для $\mathfrak{f}(31),(37)$ отсутствуют.

\section{4. ШАХМАТНАЯ МОДЕЛЬ}

Следуюшим по сложности случаем после однородной модели является случай “2-шахматной модели”. Решетка параметров $a_{\alpha, \beta}, b_{\alpha, \beta}$ и $c_{\alpha, \beta}$ имеет период 2 в обоих направлениях. Конечно же, $A$ и $B$ должны быть четными. Соответствуюшая трехмерная решеточная модель была открыта в работах [6], и ее интегрируемость следует из модифицированного уравнения тетраэдров.

Пусть

$$
\chi_{4}\left(x^{2}, y^{2}\right)=\operatorname{det}\left|\begin{array}{cccc}
1 & -x a_{1,0} & -y b_{0,1} & -x y c_{1,1} \\
-x a_{0,0} & 1 & -x y c_{0,1} & -y b_{1,1} \\
-y b_{0,0} & -x y c_{1,0} & 1 & -x a_{1,1} \\
-x y c_{0,0} & -y b_{1,0} & -x a_{0,1} & 1
\end{array}\right| .
$$

По сравнению с однородным случаем ${ }^{4}$, если $a_{\alpha, \beta}=a, b_{\alpha, \beta}=b, c_{\alpha, \beta}=c$, то

$\chi_{4}\left(x^{2}, y^{2}\right)=(1-a x-b y+c x y)(1+a x-b y-c x y)(1-a x+b y-c x y)(1+a x+b y+c x y)$.

\footnotetext{
${ }^{4)} \chi_{4}\left(x^{2}, y^{2}\right)$ может быть полностью факторизован и для более общего набора $a_{\alpha, \beta}, b_{\alpha, \beta}, c_{\alpha, \beta}$; это соответствует "неоднородной модели Замолодчикова" и в любом случае относится к нашим предыдущим вычислениям.
} 
Преобразование Фурье теперь дает

$$
J(\lambda, \mu)=\prod_{\alpha=0}^{A / 2-1} \prod_{\beta=0}^{B / 2-1} \chi_{4}\left(x^{2} e^{4 \pi i \alpha / A}, y^{2} e^{4 \pi i \beta / B}\right),
$$

где $\lambda=x^{A}, \mu=y^{B}$, а лидирующий член в $J$ в термодинамическом пределе есть

$$
\mathfrak{f}=\frac{1}{4(2 \pi)^{2}} \iint_{0}^{2 \pi} d \phi d \phi^{\prime} \ln \left|\chi_{4}\left(x^{2} e^{i \phi}, y^{2} e^{i \phi^{\prime}}\right)\right|
$$

Для упрошения рассмотрим так называемую “двухцветную” шахматную модель, т.е. случай, когда

$$
\begin{aligned}
& a_{1,1}=a_{0,0}=a_{0}, \quad b_{1,1}=b_{0,0}=b_{0}, \quad c_{1,1}=c_{0,0}=c_{0}, \\
& a_{1,0}=a_{0,1}=a_{1}, \quad b_{0,1}=b_{1,0}=b_{1}, \quad c_{1,0}=c_{0,1}=c_{1} \text {. }
\end{aligned}
$$

(Решетка параметров аналогична обычной черно-белой шахматной доске. Отметим, что в русской терминологии понятие цвета употребляется и для обозначения значений спиновых переменных, однако спиновые и шахматные цвета из разных палитр.) Теперь $\chi_{4}$ можно разложить на два неприводимых множителя:

$$
\chi_{4}\left(x^{2}, y^{2}\right)=\chi_{2}^{\prime}(x, y) \chi_{2}^{\prime}(-x, y)
$$

где

$$
\chi_{2}^{\prime}(x, y)=1-a_{0} a_{1} x^{2}-b_{0} b_{1} y^{2}+c_{0} c_{1} x^{2} y^{2}-\left(c_{0}+c_{1}+a_{0} b_{1}+a_{1} b_{0}\right) x y
$$

Без ограничения обшности можно перемасштабировать параметры

$$
a_{0} a_{1} x^{2}=\frac{u^{2}}{w^{2}}, \quad b_{0} b_{1} y^{2}=\frac{v^{2}}{w^{2}}, \quad c_{0} c_{1} x^{2} y^{2}=u^{2} v^{2}
$$

определить

$$
\chi_{2}(u, v)=1-\frac{u^{2}}{w^{2}}-\frac{v^{2}}{w^{2}}+u^{2} v^{2}-4 h \frac{u v}{w},
$$

где мы предполагаем $w \in \mathbb{R}, w>0$, и положить

$$
k^{-2}=h^{2}-\left(\frac{w-w^{-1}}{2}\right)^{2}, \quad h=\sqrt{k^{-2}+\left(\frac{w-w^{-1}}{2}\right)^{2}}
$$

Мы будем использовать краткое обозначение $\chi_{2}(u, v)$ наряду с $\chi_{2}(u, v ; w, k)$ или $\chi_{2}(u, v ; w, h)$. Однородный случай соответствует $k=1, h=\left(w+w^{-1}\right) / 2$ и

$$
\chi_{2}(u, v ; w, k=1)=\frac{(w-u-v-u v w)(w+u+v-u v w)}{w^{2}} .
$$


Теперь

$$
\mathfrak{f}=\frac{1}{2(2 \pi)^{2}} \iint_{0}^{2 \pi} d \phi d \phi^{\prime} \ln \left|\chi_{2}\left(u e^{i \phi}, v e^{i \phi^{\prime}}\right)\right| .
$$

Поскольку значение $\mathfrak{f}$ зависит от $|u|$ и $|v|$, мы можем всегда считать все $u, v, w, h$ вешественными положительными числами. Можно легко получить несколько симметрийных свойств $\mathfrak{f}$. Используя, например, $\chi_{2}(u, v, w, k)=\chi_{2}(v, u, w, k)$ или $\chi_{2}(u, v, w, k)=$ $-\left(u^{2} / w^{2}\right) \chi_{2}\left(u^{-1}, v, w^{-1}, k\right)$, можно получить

$$
\mathfrak{f}(u, v, w, k)=\frac{1}{2} \ln \frac{u v}{w}+\mathfrak{f}_{0}(u, v, w, k),
$$

где

$$
\mathfrak{f}_{0}(u, v, w, k)=\mathfrak{f}_{0}(v, u, w, k)=\mathfrak{f}\left(u^{-1}, v, w^{-1}, k\right)=\mathfrak{f}\left(u^{-1}, v^{-1}, w, k\right) .
$$

Чтобы проинтегрировать (49) по $\phi^{\prime}$, следует воспользоваться разложением

$$
\chi_{2}(u, v)=\left(1-\frac{u^{2}}{w^{2}}\right)\left(1-\lambda_{+}(u, v)\right)\left(1-\lambda_{-}(u, v)\right),
$$

где

$$
\lambda_{ \pm}(u, v)=2 \frac{u v}{w}\left(1-\frac{u^{2}}{w^{2}}\right)^{-1}\left(\sqrt{k^{-2}+\left(\frac{w-w^{-1}}{2}\right)^{2}} \pm \sqrt{k^{-2}+\left(\frac{u-u^{-1}}{2}\right)^{2}}\right) .
$$

Пусть $\phi \in \Sigma_{ \pm} \Leftrightarrow\left|\lambda_{ \pm}\left(u e^{i \phi}, v\right)\right|>1$, тогда

$$
\begin{aligned}
\mathfrak{f}= & \frac{1}{4 \pi} \int_{0}^{2 \pi} d \phi \ln \left|1-\frac{u^{2}}{w^{2}} e^{2 i \phi}\right|+ \\
& +\frac{1}{4 \pi} \int_{\phi \in \Sigma_{+}} d \phi \ln \left|\lambda_{+}\left(u e^{i \phi}, v\right)\right|+\frac{1}{4 \pi} \int_{\phi \in \Sigma_{-}} d \phi \ln \left|\lambda_{-}\left(u e^{i \phi}, v\right)\right| .
\end{aligned}
$$

4.1. Интегрирование при $u=v=1$. Для теста для фазовой структуры выберем сначала $u=v=1$. Заранее можно сказать, что в этом случае противоположные углы многоугольника Ньютона доминируют одновременно (см. замечание 3 ), и любое изменение $\mathfrak{f}$ должно быть связано с изменением основного состояния интегралов движения из середины многоугольника Ньютона для $J$.

При $u=v=1$ возникают три режима. Первый режим - это $0<k \leqslant 1$, т.е. $h \geqslant$ $\left(w+w^{-1}\right) / 2$. Тогда

$$
\mathfrak{f}_{0}(w, k)=\mathfrak{T}(w, k),
$$

где

$$
\mathfrak{T}(w, k) \stackrel{\text { пр. }}{=} \frac{1}{\pi} \int_{0}^{\pi / 2} d \phi \ln \left(2 \sqrt{k^{-2}+\left(\frac{w-w^{-1}}{2}\right)^{2}}+2 \sqrt{k^{-2}-\sin ^{2} \phi}\right) .
$$


$\mathrm{B}$ частности, при $k=1$ и $w=\operatorname{tg} \beta_{1}, 0 \leqslant \beta_{1} \leqslant \pi / 2$,

$$
\begin{aligned}
\mathfrak{T}\left(w=\operatorname{tg} \beta_{1}, k=1\right) & =\frac{2}{\pi} \text { Catalan }+\frac{1}{\pi} \int_{0}^{\pi / 2-2 \beta_{1}} \frac{\alpha d \alpha}{\cos \alpha}= \\
& =2 \Im\left(\beta_{1}\right)+2 \mathfrak{I}\left(\frac{\pi}{2}-\beta_{1}\right)-\frac{1}{2} \ln \left(2 \sin 2 \beta_{1}\right),
\end{aligned}
$$

где э определяется формулой (32), а

$$
\text { Catalan } \stackrel{\text { опр. }}{=} \sum_{j=0}^{\infty} \frac{(-)^{j}}{(2 j+1)^{2}} \sim 0.9159655942 .
$$

В этом случае $\mathfrak{f}$ совпадает с выражением (31) при $\beta_{0}=\beta_{1}$ и $\beta_{3}=\beta_{2}=\pi / 2-\beta_{1}$. Таким образом, в некотором смысле (56) - эллиптическое продолжение дилогарифма. Производная (55) (см. соотношения между $h, k, w$ в формуле (47))

$$
\frac{\partial \mathfrak{f}_{0}(w, k)}{\partial k^{-1}}=\frac{h^{-1}}{\pi} K(k)
$$

где

$$
K(k) \stackrel{\text { onp. }}{=} \int_{0}^{1} \frac{d t}{\sqrt{\left(1-t^{2}\right)\left(1-k^{2} t^{2}\right)}}
$$

имеет логарифмическую расходимость при $k \rightarrow 1$.

Второй режим - это $0 \leqslant k^{-1} \leqslant 1$, т.е $\left|w-w^{-1}\right| / 2 \leqslant h \leqslant\left(w+w^{-1}\right) / 2$. Тогда

$$
\begin{aligned}
& \mathfrak{f}_{0}(w, k)=\frac{1}{2}|\ln w|+ \\
& \quad+\frac{1}{\pi} \int_{0}^{\arcsin \left(k^{-1}\right)} d \phi \ln \left(\left[h+\sqrt{k^{-2}-\sin ^{2} \phi}\right]\left[\sqrt{\left(\frac{w-w^{-1}}{2}\right)^{2}+\sin ^{2} \phi}\right]^{-1}\right),
\end{aligned}
$$

где $0 \leqslant \arcsin \left(k^{-1}\right) \leqslant \pi / 2$, а производная

$$
\frac{\partial \mathfrak{f}_{0}(w, k)}{\partial k^{-1}}=\frac{k^{-1} h^{-1}}{\pi} K\left(k^{-1}\right) .
$$

Третий режим $-\left(w-w^{-1}\right)^{2} / 4 \leqslant-k^{-2} \leqslant 0$, т.е $0 \leqslant h \leqslant\left|w-w^{-1}\right| / 2$. Тогда

$$
\mathfrak{f}(w, k)=\frac{1}{2}|\ln w| .
$$


4.2. Интегрирование при $u, v \neq 1$. Ситуация при $u, v \neq 1$ немного иная и более сложная. Пусть $u, v, w>0$ и

$$
h_{+}=\frac{w^{2}+u^{2}+v^{2}+u^{2} v^{2} w^{2}}{4 u v w}, \quad h_{-}=\frac{w^{2}-u^{2}-v^{2}+u^{2} v^{2} w^{2}}{4 u v w} .
$$

Благодаря симметриям (51) достаточно рассмотреть случаи, когда

$$
v \leqslant u \leqslant 1
$$

Если $h_{+} \leqslant h$, то $\mathfrak{f}_{0}(u, v, w, k)=\mathfrak{T}(w, k)$ и поэтому совпадает с (55). Кандидатом на точку фазового перехода является $h=h_{+}>\left(w+w^{-1}\right) / 2$, т.е. $k_{\mathrm{cr}}^{-2} \geqslant 1$.

Область $\left|h_{-}\right| \leqslant h \leqslant h_{+}$с ограничением (63) дает $\Sigma_{+}=(-\alpha, \alpha) \cup(\pi-\alpha, \pi+\alpha)$ и $\Sigma_{-}=\varnothing$ в формальном выражении (54). Здесь $\alpha$ есть решение уравнения

$$
\left|\lambda_{+}\left(u e^{i \alpha}, v\right)\right|=1, \quad 0 \leqslant \alpha \leqslant \frac{\pi}{2} .
$$

Формально выражение для $\mathfrak{f}$ следующее:

$$
\mathfrak{f}=\frac{1}{4 \pi} \int_{0}^{2 \pi} d \phi \ln \left|1-\frac{u^{2}}{w^{2}} e^{2 i \phi}\right|+\frac{1}{2 \pi} \int_{-\alpha}^{\alpha} d \phi \ln \left|\lambda_{+}\left(u e^{i \phi}, v\right)\right| .
$$

Уравнение (64) может быть переписано как квадратное уравнение для $\cos 2 \alpha$, у которого только одно решение удовлетворяет условию $|\cos 2 \alpha| \leqslant 1$. Если $k=1$, это уравнение сводится к теореме косинусов для сферического треугольника, и параметризация типа (26) возникает естественно. Если $k \neq 1$, это квадратное уравнение неприводимо, и мы не смогли удачно запараметризовать его в терминах $u, v, w, h$. Однако можно показать, что $\partial^{2} \mathfrak{f}_{0}(u, v, w, k) / \partial k^{-2}$ сингулярна при $h \rightarrow h_{+}-0$.

Чтобы вьписать $\mathfrak{f}_{0}$ в этой области, необходимо ввести эллиптическую параметризацию. Пусть $k^{\prime}=\sqrt{1-k^{2}}$, а $K(k)$ дается формулой (58). Определения и обозначения функций Якоби и периодов можно найти в работе [12]. Для $k^{2}>0$ введем

$$
\tau(x) \stackrel{\text { опр. }}{=} \frac{k \operatorname{sn}\left(x, k^{\prime}\right)}{\operatorname{dn}\left(x, k^{\prime}\right)+\operatorname{cn}\left(x, k^{\prime}\right)}, \quad \frac{\tau^{\prime}(x)}{\tau(x)}=\sqrt{1+k^{2}\left(\frac{\tau(x)-\tau(x)^{-1}}{2}\right)^{2}}=\frac{1}{\operatorname{sn}\left(x, k^{\prime}\right)} .
$$

Обшая параметризация эллиптической кривой

$$
\chi_{2}\left(u e^{i \alpha}, v e^{-i \alpha^{\prime}}, w, k\right)=0
$$

(или, эквивалентно, $\left|\lambda_{+}\left(u e^{i \alpha}, v\right)\right|=1$ для $v<u<1$ и $k^{2}>1$ ) дается формулами

$$
u e^{i \alpha}=\tau(a+i \gamma), \quad v e^{-i \alpha^{\prime}}=\tau(b-i \gamma), \quad w=\tau(a+b),
$$

так что $u=|\tau(a+i \gamma)|, v=|\tau(b-i \gamma)|$. Условия $v<u<1$ и $w>0$ соответствуют

$$
0<a+b<2 K\left(k^{\prime}\right), \quad a>|b|, \quad 0<a<K\left(k^{\prime}\right), \quad 0<\gamma \leqslant \operatorname{Re}(K(k)) .
$$


Теперь интегрирование может быть выполнено в терминах $a, b, \gamma$ следуюшим образом (напомним, что $\mathfrak{f}_{0}=\mathfrak{f}-\ln (u v / w) / 2$ ):

$$
\begin{aligned}
\mathfrak{f}_{0}= & \frac{1}{2 \pi} \int_{\alpha}^{\pi-\alpha} d \phi \ln \left|1-\frac{u^{2}}{w^{2}} e^{2 i \phi}\right|+\left(\frac{\alpha}{\pi}-\frac{1}{2}\right) \ln \frac{u v}{w}+ \\
& +\frac{1}{2 \pi i} \int_{a-i \gamma}^{a+i \gamma} \frac{d z}{\operatorname{sn}\left(z, k^{\prime}\right)} \ln \left(2 k^{-1} \operatorname{ns}\left(a+b, k^{\prime}\right)+2 k^{-1} \mathrm{~ns}\left(z, k^{\prime}\right)\right)
\end{aligned}
$$

где $\operatorname{ns}\left(x, k^{\prime}\right) \equiv 1 / \operatorname{sn}\left(x, k^{\prime}\right)$. Это выражение преврашается в $\mathfrak{T}(w, k)$, если $\gamma=K(k)$ и $0<k<1 \Leftrightarrow \alpha=\pi / 2$ и $h=h_{+}$.

Конечно же, выражение (70) переходит в (31) при $k=1$. А именно, при $k=1$ интегрирование в (70) может быть осушествлено в терминах дилогарифмов, и для получения (31) нужно дважды применить пентагональное тождество Роджерса.

При $k^{2}<1$ и $k / i>0$ удобнее пользоваться другой параметризацией. Пусть

$$
\begin{gathered}
\sigma(x)=\tau(-i x)=\frac{k}{i} \frac{\operatorname{sn}(x, k)}{1+\operatorname{dn}(x, k)} . \\
u e^{i \alpha}=\sigma(a+i \gamma), \quad v e^{-i \alpha^{\prime}}=\sigma(b-i \gamma), \quad w=\sigma(a+b),
\end{gathered}
$$

где $0 \leqslant a<K(k), a>|b|, 0 \leqslant a+b<2 K(k)$ и $0 \leqslant \gamma \leqslant \gamma_{\mathrm{cr}}(a, b, k)$. Выражение для $\mathfrak{f}_{0}$ аналогично (70):

$$
\begin{aligned}
\mathfrak{f}_{0}= & \frac{1}{2 \pi} \int_{\alpha}^{\pi-\alpha} d \phi \ln \left|1-\frac{u^{2}}{w^{2}} e^{2 i \phi}\right|+\left(\frac{\alpha}{\pi}-\frac{1}{2}\right) \ln \frac{u v}{w}+ \\
& +\frac{1}{2 \pi i} \int_{a-i \gamma}^{a+i \gamma} \frac{d z}{\operatorname{sc}(z, k)} \ln \left(2 i k^{-1} \operatorname{cs}(a+b, k)+2 i k^{-1} \operatorname{cs}(z, k)\right),
\end{aligned}
$$

где $\operatorname{cs}(x, k) \equiv 1 / \operatorname{sc}(x, k) \equiv \operatorname{cn}(x, k) / \operatorname{sn}(x, k)$.

Для всех $k$ предел $\gamma \rightarrow 0$ (т.е. $\alpha \rightarrow 0$ ) соответствует $h \rightarrow h_{-}>0$. Ниже величины $h_{-}$ зависимость от $h$ пропадает: $\mathfrak{f}_{0}(u, v, w, h)=\mathfrak{f}_{0}\left(u, v, w, h_{-}\right)$. А именно (напомним, что $v<u<1)$

$$
\begin{aligned}
& u \leqslant w \quad \Rightarrow \quad \mathfrak{f}_{0}(u, v, w, h)=\frac{1}{2} \ln \frac{w}{u v}, \\
& w \leqslant u \quad \Rightarrow \quad \mathfrak{f}_{0}(u, v, w, h)=\frac{1}{2} \ln \frac{u}{v w}
\end{aligned}
$$

при $0<h<h_{-}$.

Специальный случай - это $h_{-}<0$ и $h<\left|h_{-}\right|$. Предел $h \rightarrow\left|h_{0}\right|$ соответствует $k^{2}<0$ и $\gamma=\gamma_{\mathrm{cr}}(a, b, k)$. Нам не удалось получить простое выражение для $\gamma_{\mathrm{cr}}$. Если $h_{-}<0$ и $h<\left|h_{-}\right|$, оба решения квадратного уравнения для $\cos (2 \alpha)$, упомянутые после формулы (64), действительны. Тогда $\Sigma_{-}$не пусто, однако мы не смогли хоть как-нибудь просто запараметризовать этот режим. 


\section{5. ОБСУЖДЕНИЕ}

Мы получили признаки нетривиальной фазовой структуры двухцветной шахматной спиновой решетки, параметризованной в терминах эллиптических интегралов. Выражения (70) и (73) показьвают важность параметризации алгебраической кривой единичного рода $\chi_{2}(u, v)=0$.

Шахматная модель является простейшим представителем класса " $M$-шахматных моделей”. Для $M$-шахматной модели структура $\left(M^{2} \times M^{2}\right)$-определителя типа (39) подразумевает униформизацию в терминах тета-функций на якобиане компактной римановой поверхности рода $g=(M-1)^{2} ; g$ модулей играют роли температуроподобных параметров (см. [8]). Таким образом, можно ожидать, что фазовая структура высших спиновых решеток весьма богата.

Благодарности. Автор выражает благодарность Г. фон Гелену и С. З. Пакуляку за множество ценных обсуждений. Работа частично финансировалась грантами INTAS OPEN 00-00055, RFBR 01-01-00201, CGP CRDF RM1-2334-MO-02.

\section{Список литературы}

[1] А. Б. Замолодчиков. ЖЭТФ. 1980. Т. 79. С. 641-664; A. B. Zamolodchikov. Commun. Math. Phys. 1981. V. 79. P. 489-505.

[2] R. J. Baxter. Commun. Math. Phys. 1983. V. 88. P. 185-205; Phys. Rev. Lett. 1984. V. 53. P. 1795-1798; Physica D. 1986. V. 18. P. 321-347.

[3] V. V. Bazhanov, R. J. Baxter. J. Stat. Phys. 1992. V. 69. P. 453-485.

[4] S. M. Sergeev, V. V. Mangazeev, Yu. G. Stroganov. J. Stat. Phys. 1996. V. 82. P. 31-50.

[5] R. J. Baxter, P. J. Forrester. J. Phys. A. 1985. V. 18. P. 1483-1497.

[6] V. V. Mangazeev, Yu. G. Stroganov. Mod. Phys. Lett. A. 1993. V. 8. P. 3475-3482; V. V. Mangazeev, S. M. Sergeev, Yu. G. Stroganov. Int. J. Mod. Phys. A. 1994. V. 9. P. 5517-5530; H. E. Boos, V. V. Mangazeev, S. M. Sergeev, Yu. G. Stroganov. Int. J. Mod. Phys. A. 1995. V. 10. P. 4041-4064.

[7] S. Sergeev. J. Phys. A. 1999. V. 32. P. 5693-5714; C. M. Сергеев. ТМФ. 2000. Т. 124. № 3. С. 391-409; Зап. научн. семин. ПОМИ. 2000. Т. 269. С. 292-307.

[8] С. З. Пакуляк, С. М. Сергеев. ТМФ. 2003. Т. 136. № 1. С. 30-51; nlin.SI/0209019.

[9] G. von Gehlen, S. Pakuliak, S. Sergeev. J. Phys. A. 2003. V. 36. P. 975-998; nlin.SI/0208035.

[10] C. М. Сергеев. ТМФ. 2004. Т. 138. № 2. C. 269-282.

[11] Ch. Fan, F. Y. Wu. Phys. Rev. B. 1970. V. 2. P. 723-733.

[12] Э. Т. Уиттекер, Дж. Н. Ватсон. Курс современного анализа. М.: Физматгиз, 1962. 